\title{
Detection of Fragmented Rectangular Enclosures in Very High Resolution Remote Sensing Images
}

\author{
Igor Zingman, Dietmar Saupe, Otávio A. B. Penatti, and Karsten Lambers
}

\begin{abstract}
We develop an approach for the detection of ruins of livestock enclosures (LEs) in alpine areas captured by highresolution remotely sensed images. These structures are usually of approximately rectangular shape and appear in images as faint fragmented contours in complex background. We address this problem by introducing a rectangularity feature that quantifies the degree of alignment of an optimal subset of extracted linear segments with a contour of rectangular shape. The rectangularity feature has high values not only for perfectly regular enclosures but also for ruined ones with distorted angles, fragmented walls, or even a completely missing wall. Furthermore, it has a zero value for spurious structures with less than three sides of a perceivable rectangle. We show how the detection performance can be improved by learning a linear combination of the rectangularity and size features from just a few available representative examples and a large number of negatives. Our approach allowed detection of enclosures in the Silvretta Alps that were previously unknown. A comparative performance analysis is provided. Among other features, our comparison includes the state-of-the-art features that were generated by pretrained deep convolutional neural networks (CNNs). The deep CNN features, although learned from a very different type of images, provided the basic ability to capture the visual concept of the LEs. However, our handcrafted rectangularitysize features showed considerably higher performance.
\end{abstract}

Index Terms-Deep features, incomplete rectangles, man-made structures, maximal cliques, object detection, rectangularity feature.

\section{INTRODUCTION}

W E ADDRESS the problem of detecting remains of manmade enclosures used to hold livestock in grassland of mountainous regions. The livestock enclosures (LEs) are of special archaeological interest because they offer important insights into historical development of alpine pastoralism [1]. Their automated spotting is the goal of a recent archaeological project [2]. Examples of such enclosures are shown in Fig. 1. These structures are usually composed of linear walls that may be heavily ruined. The most common shape of LE resembles a rectangular contour with greatly varying size and aspect ratio. Rectangle angles may deviate from right angles, and

Manuscript received December 9, 2015; revised February 25, 2016; accepted March 9, 2016.

I. Zingman and D. Saupe are with the Department of Computer and Information Science, University of Konstanz, 78464 Konstanz, Germany (e-mail: igor.zingman@uni-konstanz.de).

O. A. B. Penatti is with the Advanced Technologies Group Samsung Research Institute, 13097-160 Campinas-SP, Brazil.

K. Lambers is with the Faculty of Archaeology, Leiden University, 2333 CC Leiden, The Netherlands.

Color versions of one or more of the figures in this paper are available online at http://ieeexplore.ieee.org.

Digital Object Identifier 10.1109/TGRS.2016.2545919
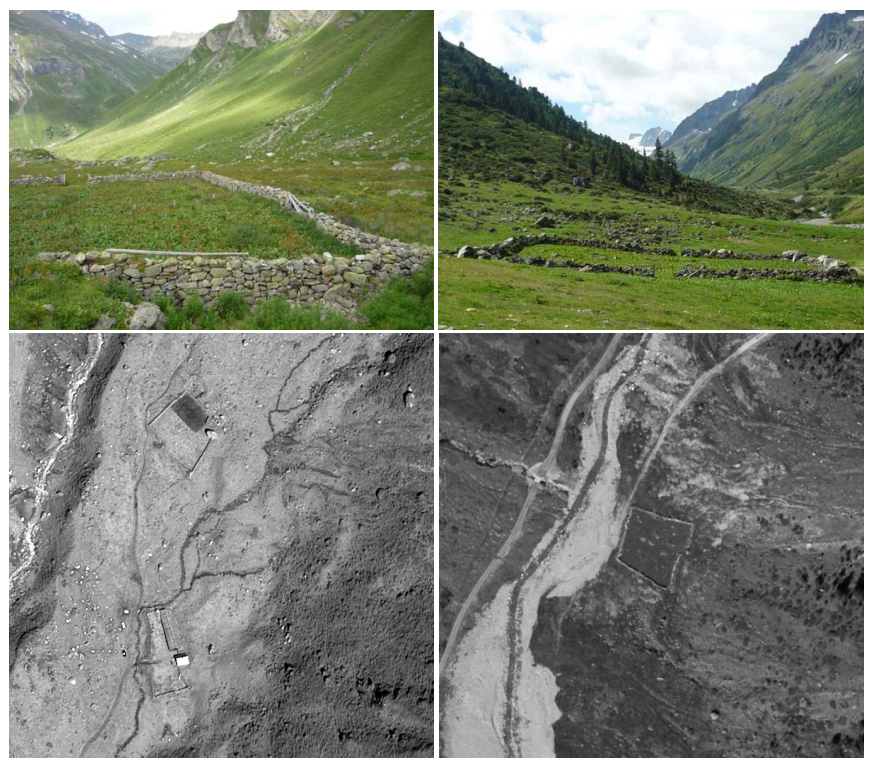

Fig. 1. (Above) LEs in alpine environment. (Below) $600 \times 600$ satellite (GeoEye 2011) and aerial (SWISSTOPO) images of $0.5-\mathrm{m}$ resolution with structures corresponding to the aforementioned LEs.

rectangle sides may be fragmented. The angle between adjacent fragments of the same side may deviate from $180^{\circ}$. Moreover, the rectangular contours are sometimes incomplete such that even an entire side may be missing.

We use satellite and aerial images of $0.5-\mathrm{m}$ resolution where the width of linear walls does not exceed two pixels. The ruined walls are of low height, which results in low-contrast linear features in the images. The spectral properties of LEs are similar to the spectral properties of the surrounding terrain, rocks, and other irrelevant objects. The second row of Fig. 1 shows a satellite and an aerial image with structures corresponding to the LEs shown previously. Nearby irrelevant structures, such as rivers, trails, or rocks, are often of similar or higher contrast either due to larger size (e.g., big rocks) or distinctive spectral properties (e.g., rivers). Detection of such faint enclosures in a complex terrain is a challenging task. Even the detection of easily modeled circular soil structures [3] had very limited success due to their low contrast and complex terrain. Only few examples of LE are available in our case, which presents another difficulty making most approaches that learn from the data inappropriate. Because of these difficulties, commonly used methods for rectangle detection are hardly applicable.

In contrast to spectral properties, the geometrical properties of LEs appear to be more distinctive and do not depend on image modality and conditions under which an image was 


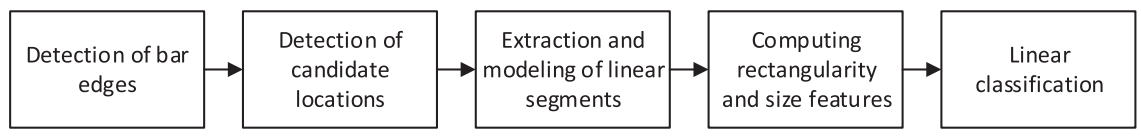

Fig. 2. Algorithmic steps for detection of approximately rectangular enclosures.

captured. We therefore develop a measure that quantifies the distinctive geometry of approximately rectangular enclosures. Our approach relies on a new rectangularity feature that discriminates rectangular patterns from other structures in a complex cluttered background. The feature is based on a prior model of a fragmented rectangle, which is a convex polygon with constrained angles.

\section{A. Related Work}

Detection of rectangular structures has previously been addressed in different contexts. Examples are detection of buildings in remotely sensed images [4]-[14], traffic signs [15]-[17], and particles of a rectangular shape in cryo-electron microscopy images [18], [19]. The methods used were based on Markov random fields (MRFs) [9], [15], marked point processes (MPPs) [10], [14], search on a graph [6], [20], Hough transform and other voting schemes [7], [8], [16]-[18], template matching [21], aggregation of local features [10], [11], [13], and heuristic rules [5].

Most techniques for detection of rectangular structures dealt with buildings in remotely sensed images. For example, in the graph-based approach in [6], a search for cycles was used to generate building hypotheses. The search was accompanied by an extensive set of rules and thresholds, which limits the robustness of the approach. MRFs were used in [9] to delineate buildings. More recently, a similar approach has been used in [15] for detection of traffic signs in color images. The approach is sensitive to inaccuracy of extracted edges and cannot detect incomplete rectangles, as it requires the presence of all four sides of a rectangular structure. The MPPs [22] have recently become popular for extraction of various structures in remotely sensed images, including buildings (e.g., in [10] and [14]). The MPP proved to be very powerful when applied to real data. However, these stochastic methods are still computationally expensive. Similarly to the MRF, they may not converge to a globally optimal solution and usually need careful tuning of a large number of parameters. Attempts have recently been made to address some of these problems, which are crucial for the analysis of large images. In [23], substantial improvements in performance have been achieved for the extraction of line networks (roads and rivers). In this paper, also the potential of GPUs was efficiently exploited.

An approach for the detection of rectangular contours based on the Hough transform was developed in [8]. The approach relies on certain strict geometrical rules, making it not suitable for detection of fragmented or incomplete structures. It may also result in detection of rectilinear configurations that cannot form a rectangular contour. Detection of such configurations is prevented in our approach by adding a convexity constraint.

In [11], a set of local features that carried local corner information was used to produce a probability map of building rooftops. Unfortunately, in the case of fragmented enclosures, corners are not reliable features. Moreover, local features, in general, do not suffice in the case of faint contours appearing in a cluttered background. A more global description that takes into account spatial relations between local features is necessary. For example, in [10] and [13], the gradient orientation density function (GODF) was computed from image gradients. A correlation of this function with a mixture of two Gaussians having mean values separated by $90^{\circ}$ served as a GODF-based feature indicating the presence of buildings.

Although there is a variety of methods developed for building detection, they are not applicable to our task because buildings are much more salient structures. In contrast to building rooftops, walls of ruined LEs are narrow and are of low height (low-contrast features), may be highly fragmented, or are even completely missing. Higher contrast irrelevant structures may appear inside or outside of rectangular structures in the immediate neighborhood. Various cues (rooftop color, shadows, 3-D cues, etc.) usually employed in building detection algorithms are not available.

\section{B. Overview of Our Approach}

Our approach follows the diagram in Fig. 2, which is briefly described as follows. A binary map of bar edges accompanied by angle information is computed first. The junction points of the medial axis of an inverted binary edge map are detected as candidate points (Section II), and a region within an analysis window centered at each candidate point is inspected. A windowed Hough transform is used to find linear segments and model them with a few parameters (Section III-A). An undirected graph is then constructed, the nodes of which correspond to linear segments, and graph edges encode spatial relations between linear segments. In particular, we use angle and convexity properties to encode spatial relations (Section III-B). Due to the construction of the graph, its maximal cliques correspond to valid configurations of linear segments. The valid configurations are then ranked by a new rectangularity measure that encodes the goodness of grouping the segments into a rectangular structure (Section III-C). In contrast to [20], the new rectangularity measure does not rely on a heuristic partitioning of the set of linear segments into four subsets. Hard decisions are softened. Configurations better matching a rectangular structure result in a higher rectangularity measure. The rectangularity feature is introduced in Section III-D. It is defined as the maximal rectangularity measure of all valid configurations. In practice, the low number of corresponding maximal cliques within the analysis window allows exact maximization, which can efficiently be computed. The resulting rectangularity feature captures the presence of $\Pi$-like structures and is robust to their fragmentation. In Section IV, we show how to improve the detection performance based solely on 
the rectangularity feature by introducing an additional feature proportional to enclosure size and learning the optimal feature combination from a large number of negative examples and just few positives. In our application, we complete the core algorithmic steps summarized in Fig. 2 with a preprocessing stage in the beginning that filters out irrelevant regions (Section V), e.g., texture regions, and with a final detector at the end, e.g., a simple thresholding (Section IV).

This paper follows our work presented in [24]. Here, we give a more detailed description of the methods, design a more efficient detector of initial candidate locations (Section II), report on the results of application of our approach to a large region in the Silvretta Alps (Section V), and extend our experimental part (Section VI) by comparing the discrimination ability of the introduced and alternative features for our task. In particular, we evaluate the performance of the features generated by several pretrained deep convolutional networks [25]-[29], the histogram of oriented gradients (HOG) [30], as well as 1-D features, such as the GODF-based feature [10] designed for building detection, and the normalized maximal rectangularity (NMR) measure [20] that we developed earlier for the detection of the LEs. We conclude in Section VIII with the discussion on shortcomings and advantages of the rectangularity and deep features for our problem.

\section{Detection of CANDidate Locations}

As in [20], we detect candidate locations from a map of bar edges (ridges and valleys) that were extracted using the morphological feature contrast (MFC) line detector [31], [32]. This technique extracts linear features while suppressing texture elements of cluttered background. We also experimented with other approaches [33]-[35], but these are either not sensitive enough to extract faint edges of enclosures or generate lots of clutter edges depending on the parameters used. The parameterless line segment detector of [36], which is known to provide robust results for a large range of images, misses faint edges of ruined enclosures.

In [20], the candidate points were obtained by sampling the medial axis of an inverted binary edge map. The medial axis was obtained by thinning the inverted edge map. The number of candidates was determined by the choice of the sampling rate. Decreasing the sampling rate reduces the number of candidates, which speeds up further validation. However, this may result in the loss of promising candidates. Instead, we suggest here to look for medial axis junction points only, which are yielded by at least three sides of an enclosure structure. This greatly reduces the number of candidates without the risk of losing enclosures with at least three remaining sides.

In [37]-[39], the medial axis of a shape was extracted by thresholding the average flux of the gradient field of the Euclidean distance function $D$ to the boundary of the shape. The average flux of the gradient field through the boundary $\partial \mathcal{N}$ of a region is defined as the corresponding flux normalized by the length of the boundary

$$
F(\nabla D)=\frac{\oint_{\partial \mathcal{N}} \nabla D \cdot \mathbf{n} d L}{\oint_{\partial \mathcal{N}} d L}
$$

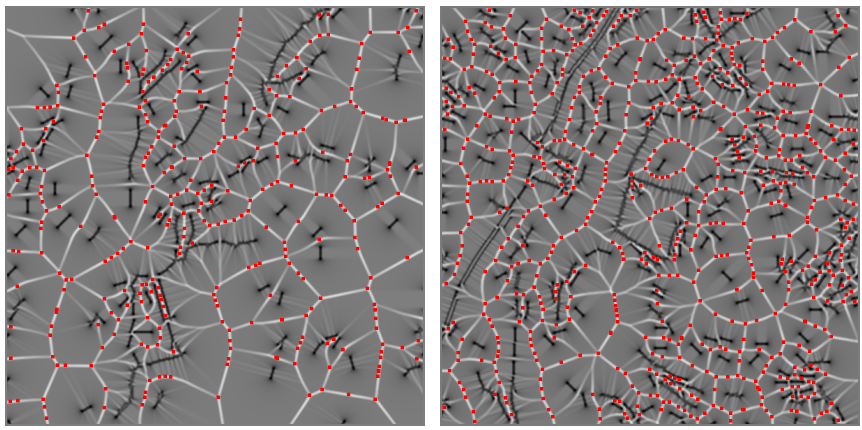

Fig. 3. Average flux of the gradient field of the distance function computed for the edge maps of the images in Fig. 1. The medial axis coincides with positive singularities of the flux (white), while edges coincide with negative singularities (black). Local maxima (red points) are used as candidate locations. Best viewed in digital version.

where $\mathbf{n}$ denotes the inward normal ${ }^{1}$ to the boundary $\partial \mathcal{N}$ and $d L$ is the boundary element. As the region $\mathcal{N}$ shrinks to a point, the average flux $F$ approaches zero at nonmedial points and nonzero values at the medial axis of the shape.

We detect candidate points by finding the local maxima of a discrete approximation of the average flux $F(\nabla D)$ through the boundary of a small disk $\mathcal{N}$, where $D$ is the distance function of the binary edge map. These local maximum points usually correspond to the junction points of the medial axis of the inverted binary edge map. Only the local maxima with the average flux greater than 0.5 were taken into account. Fig. 3 shows the examples of detections (in red) overlaid on the average flux, which has positive extrema on the medial axis (white) and negative extrema on the bar edges (black). ${ }^{2}$

In a related approach [40], nonmaxima suppression was applied to the average flux of the normalized gradient vector flow (GVF) [41], [42] in order to detect medial feature points. Using the GVF instead of the gradient field of the distance transform of the edge map allowed detection of medial feature points directly from the grayscale image without the need of edge extraction. However, GVF may ignore weak gradients of low-contrast structures of our interest. In addition, computing GVF might be too slow on large images, depending on the number of predefined iterations.

We combine candidate points separately obtained from the binary maps of ridge and valley edges. The structures that are within a window around the candidate points $p$ are further analyzed. The size of the analysis window can be determined adaptively, based on the value of the distance transform $D(p)$, i.e., the distance of $p$ to a candidate structure. Similarly to [20], we use the circular analysis window of radius $D(p) \sqrt{a^{2}+1}$ centered at $p$ that circumscribes a rectangle centered at $p$ with (small) side length $2 D(p)$ and aspect ratio $a$. In our experiments, $a$ was set to 1.4 . We discarded all candidate points having a distance $D$ less than 15 or greater than 90 pixels, which limits the distances between opposite walls of the structures to be in between 7.5 and $45 \mathrm{~m}$.

\footnotetext{
${ }^{1}$ In [37]-[39], the outward normal was used.

${ }^{2}$ We used valley edges for the left figure and ridge edges for the right figure.
} 


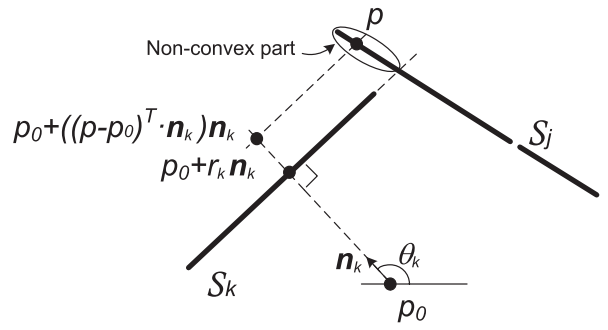

Fig. 4. Fraction of points $p$ of $\mathcal{S}_{j}$ that violates the convexity constraint relative to $\mathcal{S}_{k}$ and $p_{0}$ is given by $\tilde{\tau}_{k, j}$. Note that linear segments can be fragmented, having small gaps as in $\mathcal{S}_{j}$.

\section{Measuring Structure Rectangularity}

We introduce a rectangularity feature $f_{R}$ computed from a set of linear segments $\mathbf{W}=\left\{\mathcal{S}_{i}, i=1, \ldots, m\right\}$ that were extracted from a grayscale image.

\section{A. Grouping Edge Points Into Linear Segments}

Given a candidate location and edge points accompanied by estimated orientations, we extract and parameterize linear segments, each of which is a group of aligned edge points. Linear segments are represented by a triple of parameters $(\theta, r, l)$ found by the use of a local Hough transform centered at the candidate points. We use the Hough transform in the form introduced in [43], where a line is defined by the orientation $\theta$ of the normal and a distance $r$ from the origin

$$
r=x \cos \theta+y \sin \theta .
$$

The spatial coordinates of an edge point are $x, y$. We use the parameterization $\theta \in[0,360)$ and $r \in(0, \infty)$ of a Hough plane. A peak at $(\theta, r)$ in the Hough plane corresponds to a line. The peaks are detected as regional maxima in the Hough plane that was discretized with $\Delta \theta=3^{\circ}$ and $\Delta r=1$ pixel. The detected line corresponds to either a single connected linear segment $\mathcal{S}$ or to several aligned connected components. In the latter case, the connected components with gaps smaller than a predefined threshold (3 pixels in our experiments) are considered as a single linear segment (see the segment $\mathcal{S}_{j}$ in Fig. 4); otherwise, they are considered as separate linear segments. The parameter $l$ in the triple $(\theta, r, l)$ is the number of points that belong to the linear segment. To better relate the parameter $l$ to the length and avoid its dependence on the width of the extracted edges, we perform their thinning [44] prior to clustering in a Hough plane.

Since edges were extracted together with their orientations, $r$ can be directly computed for each edge point $(x, y)$ using (2). Thus, each edge point votes for a single point in the $(\theta, r)$ plane instead of voting for a curve as suggested in [43]. This idea, which was used already in [45] for clustering of short ridge features, considerably eases extraction of meaningful peaks in the Hough plane.

\section{B. Valid Configurations of Linear Segments}

In the following, we define a valid configuration of linear segments $\mathbf{C} \subseteq \mathbf{W}$ that can be a part of a rectangular structure.
We require angles $\beta_{k, j}$ between linear segments $\mathcal{S}_{k}, \mathcal{S}_{j} \in \mathbf{C}$ to be close to either zero, $180^{\circ}$, or right angles. An angle tolerance $\alpha$ will be set to control the strictness of the angle constraint. We define $\beta_{k, j}$ as

$$
\beta_{k, j}=\min \left(\left|\theta_{\mathcal{S}_{k}}-\theta_{\mathcal{S}_{j}}\right|, 360-\left|\theta_{\mathcal{S}_{k}}-\theta_{\mathcal{S}_{j}}\right|\right) .
$$

Note that $\beta_{j, k}=\beta_{k, j}$ and $\beta \in[0,180]$, since $\theta \in[0,360)$.

The angle constraint alone does not suffice to restrict configurations to be perceptually close to rectangles or rectangle parts. We therefore define a second constraint that requires the valid configuration to be nearly convex in the sense that extension of all linear segments of the configuration can form a nearly convex contour. The convexity tolerance $t$ will be defined to control the strictness of the convexity constraint. For a convex configuration of linear segments, it is required that a half plane generated by each segment includes all other segments of the configuration. Additionally, we require all of these half planes to contain the candidate point around which we search for a rectangular structure. Pairwise convexity constraints suffice to verify the convexity of a configuration containing the given candidate point. We define the pairwise convexity measure $\tau$ for a pair of linear segments $\mathcal{S}_{k}, \mathcal{S}_{j}$, each with corresponding attributes of size $l_{\mathcal{S}}$, orientation $\theta_{\mathcal{S}}$, and distance $r_{\mathcal{S}}$ to the candidate point $p_{0}$, as

$$
\begin{aligned}
\tau_{k, j} & =\max \left(\tilde{\tau}_{k, j}, \tilde{\tau}_{j, k}\right) \\
\tilde{\tau}_{k, j} & =\frac{1}{l_{j}} \sum_{p \in \mathcal{S}_{j}} H\left(\left(p-p_{0}\right)^{T} \cdot n_{k}-r_{k}\right)
\end{aligned}
$$

where $n_{k}=\left(\cos \theta_{k}, \sin \theta_{k}\right)^{T}$ is the unit normal of $\mathcal{S}_{k}$ and $H(u)$ is an indicator function equal to 1 for $u>0$ and 0 otherwise. $\tilde{\tau}_{k, j}$ measures the relative number of points in the segment $\mathcal{S}_{j}$ that are behind the segment $\mathcal{S}_{k}$, relative to the given candidate point $p_{0}$ as illustrated in Fig. 4. Note that $\tau \in[0,1]$, and $\tau_{k, j}=$ $\tau_{j, k}$, while $\tilde{\tau}_{k, j} \neq \tilde{\tau}_{j, k}$.

Definition 1: Let $\alpha \in[0,45], t \in[0,1]$, a candidate point $p_{0}$, and a configuration $\mathbf{C}$ of linear segments be given. If for all pairs $\mathcal{S}_{k}, \mathcal{S}_{j} \in \mathbf{C}, j \neq k$, one of the inequalities of the angle constraint

$$
\beta_{k, j} \leq \alpha \text { or }\left|90-\beta_{k, j}\right| \leq \alpha \text { or } 180-\beta_{k, j} \leq \alpha
$$

and the convexity constraint

$$
\tau_{k, j} \leq t
$$

both hold, then $\mathbf{C}$ is called a $(t, \alpha)$-valid configuration located around $p_{0}$ and denoted by $\mathbf{C}_{p_{0}}^{t, \alpha}$.

For the sake of brevity, we usually omit the indices $t$, $\alpha$ and the reference point $p_{0}$, mentioning that $\mathbf{C}$ is a valid configuration. Valid configurations include not only perfect rectangles but also convex polygons or their parts with angles around either $90^{\circ}$ or $180^{\circ}$. This is important in practice since approximately rectangular structures are better modeled by such polygons rather than by perfect rectangles. 


\section{Rectangularity Measure of a Valid Configuration}

A couple of poorly aligned short segments can be a valid configuration as far as the tolerances $t, \alpha$ allow. There is a need to rank valid configurations according to their similarity to a canonical rectangle. To find and rank valid configurations, we construct an undirected graph $\mathbf{G}^{w}$ from the given set $\mathbf{W}$ of linear segments in a window centered at a candidate point $p_{0}$. The graph $\mathbf{G}^{w}$ has nodes $j=1, \ldots, m$ corresponding to the segments $\mathcal{S}_{1}, \ldots, \mathcal{S}_{m} \in \mathbf{W}$. Each node $j$ is attributed by a triple of parameters $\left(\theta_{j}, r_{j}, l_{j}\right)$, i.e., orientation, distance to the reference point $p_{0}$, and size of the linear segment. An edge $\{k, j\}$ is attributed with the angle $\beta_{k, j}$ and the pairwise convexity $\tau_{k, j}$ of the corresponding pair of segments $\mathcal{S}_{k}, \mathcal{S}_{j}$. An edge $\{k, j\}$ is included in the graph $\mathbf{G}^{w}$ if $\beta_{k, j}$ and $\tau_{k, j}$ satisfy the constraints in (6) and (7). This attributed graph encodes the properties of the linear segments and their spatial relationships. Due to the graph construction and Definition 1, valid configurations $\mathbf{C}$ correspond to fully connected subgraphs $\mathbf{G}^{c}$, also called cliques, of the graph $\mathbf{G}^{w}$.

In the following, we introduce the new rectangularity measure $\rho\left(\mathbf{G}^{c}\right)$ that ranks a clique $\mathbf{G}^{\mathbf{c}}$ corresponding to a valid configuration $\mathbf{C} \subseteq \mathbf{W}$. We define the measure with the following properties in mind. The rectangularity measure shall yield higher values for configurations with

1) higher degree of convexity given by lower values of the convexity measure $\tau$;

2) higher degree of angle alignments given by angles $\beta$;

3) longer linear segments given by larger $l$.

In addition, the proposed rectangularity measure shall

4) have the increasing property $\rho\left(\mathbf{G}_{1}^{c}\right) \leq \rho\left(\mathbf{G}_{2}^{c}\right)$ for $\mathbf{G}_{1}^{c} \subseteq$ $\mathbf{G}_{2}^{c}$. Thus, the rectangularity measure of a larger encompassing clique has a higher value.

5) yield a zero value for configurations of linear segments with less than three sides of a rectangle.

We define the rectangularity measure of a graph clique $\mathbf{G}^{c}$ in terms of sums over its undirected edges $\{k, j\} \in E^{c}$

$$
\begin{aligned}
\rho\left(\mathbf{G}^{c}\right)= & \left(\left(\sum_{\{k, j\} \in E^{c}} l_{k} l_{j} f_{90}\left(\beta_{k, j}\right) f_{c v}\left(\tau_{k, j}\right)\right)\right. \\
& \left.\times\left(\sum_{\{k, j\} \in E^{c}} l_{k} l_{j} f_{180}\left(\beta_{k, j}\right) f_{c v}\left(\tau_{k, j}\right)\right)\right)^{\frac{1}{4}}
\end{aligned}
$$

where $f_{90}, f_{180}$, and $f_{c v}$ are mode functions depicted in Fig. 5 . $f_{90}$ and $f_{180}$ are equal to zero for angles $\beta$ that deviate from the mode center larger than the angle tolerance $\alpha . f_{c v}$ is equal to zero for the convexity measure $\tau$ larger than the convexity tolerance $t$. In our experiments, we used $\alpha=35^{\circ}$ and $t=0.3$. The exact definition of the mode function is not critical and is not given here due to space considerations.

The first factor of $\rho\left(\mathbf{G}^{c}\right)$ in (8) yields a nonzero value only if the valid configuration $\mathbf{C}$ contains at least one pair of approximately perpendicular linear segments that fulfill the convexity constraint in (7). The second factor is nonzero only
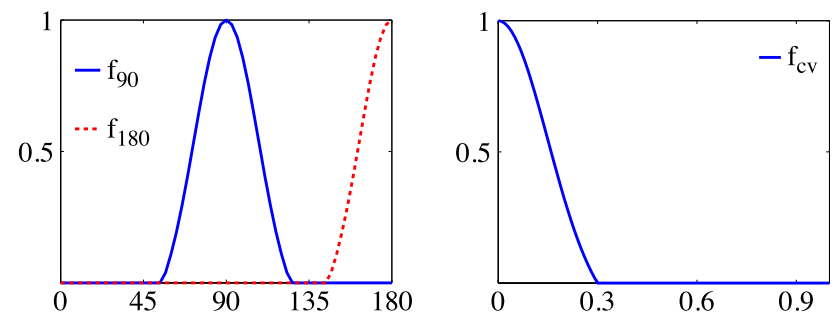

Fig. 5. Functions $f_{90}$ (left figure, solid blue curve), $f_{180}$ (left figure, dashed red curve), and $f_{c v}$ (right figure) used in the rectangularity measure in (8).

if the valid configuration contains at least one pair of approximately parallel linear segments. ${ }^{3}$ The product of these two factors is nonzero only if the valid configuration $\mathbf{C}$ contains at least one pair of parallel and one pair of perpendicular linear segments. The angles between linear segments of these parallel and perpendicular pairs are restricted to be approximately $0^{\circ}$, $180^{\circ}$, or $90^{\circ}$ since $\mathrm{C}$ is a valid configuration with linear segments constrained by (6). Thus, a nonzero rectangularity measure ensures a valid configuration $\mathbf{C}$ containing at least one triple of segments arranged in a П-like structure, as stated in property 5 previously. This property allows suppression of a large number of configurations originating from clutter (e.g., lines, corners, junctions, etc.). It is easy to verify that the other four aforementioned properties are also satisfied by the rectangularity measure in (8). Also note that the rectangularity measure scales linearly with the spatial size of rectangles.

\section{Rectangularity Feature}

Given a set of linear segments $\mathbf{W}$ in an analysis window, we define the rectangularity feature $f_{R}$ of the corresponding graph $\mathbf{G}^{w}$. We denote the set of cliques of $\mathbf{G}^{w}$ as $\mathcal{K}\left(\mathbf{G}^{w}\right)$. The rectangularity feature of $\mathbf{G}^{w}$ is defined as

$$
f_{R}\left(\mathbf{G}^{w}\right)=\max _{\mathbf{G}^{c} \in \mathcal{K}\left(\mathbf{G}^{w}\right)} \rho\left(\mathbf{G}^{c}\right) .
$$

The corresponding optimal clique is

$$
\mathbf{G}_{\mathrm{opt}}^{c}=\underset{\mathbf{G}^{c} \in \mathcal{K}\left(\mathbf{G}^{w}\right)}{\arg \max } \rho\left(\mathbf{G}^{c}\right) .
$$

Due to the increasing property of $\rho$ (the fourth property of the rectangularity measure stated in Section III-C), the maximum can be searched over the set of maximal cliques ${ }^{4}$ only, denoted here by $\mathcal{M}\left(\mathbf{G}^{w}\right)$. That is

$$
f_{R}\left(\mathbf{G}^{w}\right)=\rho\left(\mathbf{G}_{\mathrm{opt}}^{c}\right)=\max _{\mathbf{G}^{c} \in \mathcal{M}\left(\mathbf{G}^{w}\right)} \rho\left(\mathbf{G}^{c}\right) .
$$

Since the set of maximal cliques $\mathcal{M}\left(\mathbf{G}^{w}\right) \subseteq \mathcal{K}\left(\mathbf{G}^{w}\right)$ is much smaller than the set of graph cliques $\mathcal{K}\left(\mathbf{G}^{w}\right)$, the number of times the rectangularity measure $\rho$ needs to be evaluated in (11) is considerably reduced in comparison to (9). Since, in addition,

\footnotetext{
${ }^{3} f_{c v}$ in the second term has only a small impact on results. It reduces the rectangularity measure for configurations with badly aligned opposite sides with a nonzero convexity measure.

${ }^{4}$ Maximal cliques are cliques that are not contained in larger cliques.
} 

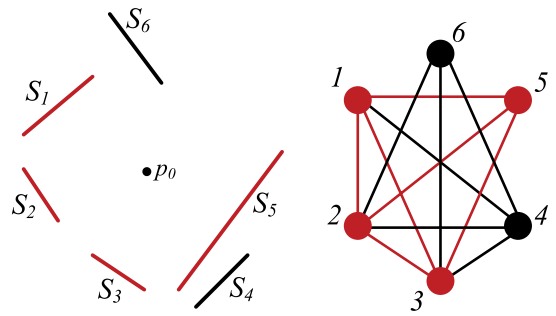

Fig. 6. (Left) Set $W=\left\{\mathcal{S}_{1}, \mathcal{S}_{2}, \ldots, \mathcal{S}_{6}\right\}$ of linear segments around a candidate point $p_{0}$. (Right) Graph $\mathbf{G}^{w}$ for the set of linear segments. We assume an angle tolerance $\alpha$ such that all angle constraints are satisfied. Several node pairs of the graph are not connected by an edge due to the convexity constraint, which is not satisfied for an assumed convexity tolerance $t$. The red nodes of the graph are the nodes of the optimal maximal clique $\mathbf{G}_{\mathrm{opt}}^{c}$. The corresponding valid configuration $\mathbf{C}_{\text {opt }}$ is marked in red on the left figure.
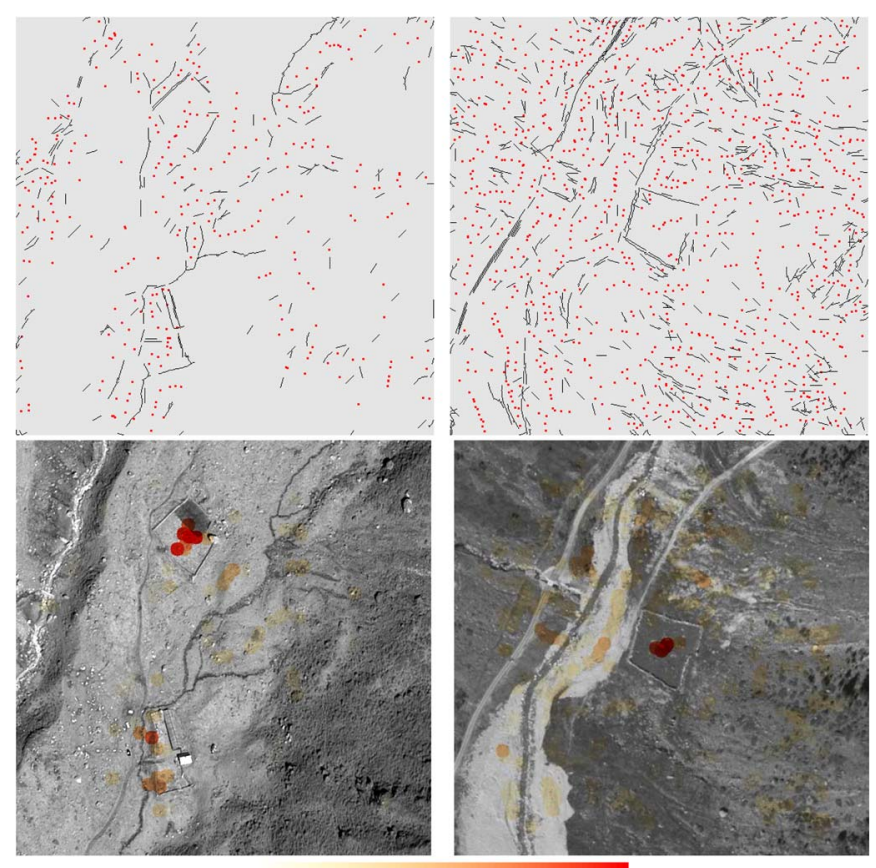

Fig. 7. (First row) Bar edges (black) and candidate points (red) generated from the images in Fig. 1. (Second row) The rectangularity feature computed at each candidate point and visualized by a colored disk. Color saturation increases, and hue is changing from yellow to red for growing values of the features in accordance with the color bar at the bottom.

there are efficient algorithms for finding maximal cliques, e.g., [46], we compute the rectangularity feature by an exhaustive search for the maximum in (11).

Fig. 6 (left) shows an example of a given set $W=\left\{\mathcal{S}_{1}, \mathcal{S}_{2}\right.$, $\left.\ldots, \mathcal{S}_{6}\right\}$ of linear segments and the optimal configuration $\mathbf{C}_{\text {opt }}=\left\{\mathcal{S}_{1}, \mathcal{S}_{2}, \mathcal{S}_{3}, \mathcal{S}_{5}\right\}$ in red, while Fig. 6 (right) shows the corresponding graph $\mathbf{G}^{w}$ and the optimal maximal clique $\mathbf{G}_{\mathrm{opt}}^{c}$ in red. There are two additional maximal cliques $\mathbf{G}_{1}^{c}$ and $\mathbf{G}_{2}^{c}$ and corresponding valid configurations $\mathcal{C}_{1}=\left\{\mathcal{S}_{2}, \mathcal{S}_{3}, \mathcal{S}_{4}, \mathcal{S}_{6}\right\}$ and $\mathcal{C}_{2}=\left\{\mathcal{S}_{1}, \mathcal{S}_{2}, \mathcal{S}_{3}, \mathcal{S}_{4}\right\}$. They, however, have lower rectangularity values $\rho\left(\mathbf{G}_{1}^{c}\right)<\rho\left(\mathbf{G}_{\text {opt }}^{c}\right), \rho\left(\mathbf{G}_{2}^{c}\right)<\rho\left(\mathbf{G}_{\text {opt }}^{c}\right)$.

Fig. 7 shows a couple of examples of the rectangularity feature computed for the real satellite and areal images. The first row shows detected bar edges and candidate points ${ }^{5}$ (Section II). The rectangularity feature $f_{R}$ computed at the candidate points is visualized by colored disks in the second row. As expected, high values were obtained at positions of LE, while zero or low values were obtained at most other candidate positions. One can see that the rectangularity feature map is quite sparse. This is partially because the rectangularity feature has a zero value for spurious structures with less than three sides.

\section{LEARNING IN THE RECTANGULARITY-SizE FeATURE SpaCe}

The rectangularity feature scales with the structure size having lower values for small structures. A detector based on such a feature is prone to dismiss small rectangles. On the other hand, false structures of a small size are more frequent. We therefore introduce an additional feature $f_{S}$ proportional to the structure size and learn a classifier from the available data in the 2-D rectangularity-size feature space. This may improve the tradeoff between the sensitivity and the number of false detections in comparison to the 1-D case. We define the size of the structure, represented by the optimal clique $\mathbf{G}_{\mathrm{opt}}^{c} \subseteq \mathbf{G}^{w}$, as

$$
f_{S}\left(\mathbf{G}^{w}\right)=\frac{\sum_{j} l_{j} r_{j}}{\sum_{j} l_{j}}
$$

where the sums are over all nodes of the optimal clique $\mathbf{G}_{\mathrm{opt}}^{c}$. $f_{S}$ is computed as the weighted distance of the linear segments of $\mathbf{C}_{\text {opt }}$ from the corresponding candidate point, where the weights are segment sizes.

Since only a few positive examples are available in our case, a classification approach should be carefully chosen. The linear classifiers are favorable when there is a danger of overfitting the data due to a limited number of available examples. They also are not computationally demanding. The normal $w$ of the separating hyperplane of a linear classifier can be found by means of the Fisher linear discriminant analysis (FLD). In this approach, the optimal direction is determined such that the data from two classes projected on $w$ are maximally separated. The separation is measured by the squared distance between class means normalized by the sum of their variances [45], [47]. This approach results in a simple solution represented in terms of class means and covariance matrices. In our case, however, the number of positive examples is very limited, and the covariance matrix cannot reliably be estimated.

We optimize the normal direction $w$ based on the large number of available samples from the dominant class of negatives and just a few examples from the class of positives. Let us define the expected signed distance between a deterministic point $y$ (positive example) and the distribution $X$ of negatives,

\footnotetext{
${ }^{5}$ Note that not all of the candidate points are the same as in Fig. 3. In contrast to Fig. 3, the map of candidate points in Fig. 7 resulted from the union of points coming from both valley and ridge edges. On the other hand, candidate points that are too distant or too close to the edges were removed (see Section II), and they do not appear in Fig. 7.
} 
both projected to the direction $w$ and normalized by the standard deviation of the projected distribution

$$
D_{w}(y, X) \equiv \frac{E_{x}\left[w^{T} y-w^{T} x\right]}{\sqrt{E_{x}\left[\left(w^{T} x-w^{T} \mu_{x}\right)^{2}\right]}}=\frac{w^{T}\left(y-\mu_{x}\right)}{\sqrt{w^{T} C_{x} w}}
$$

where $\mu_{x}$ and $C_{x}$ are the mean and the covariance matrix of the distribution $X$, respectively. Next, we define the average signed distance between a set of deterministic points $\left\{y_{i}, i=\right.$ $1, \ldots, n\}$ and the distribution $X$

$$
\bar{D}_{w}\left(\left\{y_{i}\right\}, X\right) \equiv \frac{1}{n} \sum_{i=1}^{n} D_{w}\left(y_{i}, X\right)=\frac{w^{T}\left(\bar{y}-\mu_{x}\right)}{\sqrt{w^{T} C_{x} w}}
$$

where $\bar{y}=(1 / n) \sum_{i=1}^{n} y_{i}$. We now define the optimal direction $w$ as the direction that maximizes the absolute value of the average signed distance between a set of points corresponding to positive examples and the distribution of the dominant class of negatives $X$, i.e.,

$$
w_{\text {opt }} \equiv \underset{w}{\arg \max }\left|\bar{D}_{w}\left(\left\{y_{i}\right\}, X\right)\right| .
$$

From (14) and (15), we obtain

$$
w_{\text {opt }}=\underset{w}{\arg \max } \frac{\left|w^{T}\left(\bar{y}-\mu_{x}\right)\right|}{\sqrt{w^{T} C_{x} w}} .
$$

It can be shown that

$$
w_{\mathrm{opt}}=C_{x}^{-1}\left(\bar{y}-\mu_{x}\right)
$$

is a solution of (16). The obtained direction $w_{\text {opt }}$ is similar to the one in the FLD analysis [45]. In contrast to the FLD solution, (17) includes the covariance matrix of the class of negatives only, preferring the solution in the direction of the small variance of negatives. Negatives are well sampled in our problem, and their covariance matrix can be robustly estimated. The positives are treated as deterministic points in the feature space and influence the solution only via their average. Literally, the average only weakly guides the solution pointing to the relevant location in the feature space. Note that the signed distance in (14) for $w_{\text {opt }}$ given in (17) yields a positive value equal to the Mahalanobis distance $\bar{D}_{w_{\text {opt }}}\left(\left\{y_{i}\right\}, X\right)=$ $\sqrt{\left(\bar{y}-\mu_{x}\right)^{T} C_{x}^{-1}\left(\bar{y}-\mu_{x}\right)}$ with the metric $C_{x}$.

The samples of $X$ may include outliers. Therefore, in (17), we use the robust multivariate trimming (MVT) estimates of the mean and the covariance matrix [48]. The MVT is an iterative technique with mean and covariance matrices recomputed at each iteration. Given the current estimates of $\mu_{x}$ and $C_{x}$, the Mahalanobis distance is computed for all of the data points. A specified percentage of the observations with the largest Mahalanobis distance is discarded, and the remaining data are used to recompute the estimates of $\mu_{x}$ and $C_{x}$. The technique is initialized with the sample mean and covariance matrix computed from the whole data. The samples with zero rectangularity $f_{R}$, which correspond to nonvalid configurations, were excluded from such a training procedure. In our experiments, we used three iterations and discarded $10 \%$ of observations in each iteration.
We will refer to

$$
f_{\mathrm{RS}}=\left(\begin{array}{c}
f_{S} \\
f_{R}
\end{array}\right)
$$

as the rectangularity-size features. Given the optimal direction $w_{\text {opt }}$, the LE structures are detected by

$$
f_{\mathrm{RS}}^{T} w_{\mathrm{opt}}>b
$$

where $b$ is a threshold to be set. It determines the tradeoff between the sensitivity and the rate of false detections. The optimal linear feature combination $f_{\mathrm{RS}}^{T} w_{\mathrm{opt}}$, which is computed at candidate points, can be seen as a confidence measure of an enclosure structure being present in the area around the candidate point. Note that learning the optimal feature combination $w_{\mathrm{opt}}$ as shown previously is not limited to 2-D feature spaces but directly extends to higher dimensions. This will be used in our experiments in Section VI in order to compare the developed features with high-dimensional generic features.

Somewhat similar ideas of using linear discriminant analysis (LDA) adapted to a small number of positives within the context of pedestrian detection appeared in [49]. Relying on the highdimensional HOG features [30] and LDA, the authors modeled the background class with the mean and covariance matrix learned from unlabeled image patches. Their model trained on a few positives was highly competitive. In contrast to [49], our model was explicitly derived from the optimization of (15) that was defined as a way to cope with the settings of the highly unbalanced problem at hand.

\section{Detection of EnClosures: the SilvRetTa Alps Case StUdy}

We built a user interface that allows a user to explore large images, shows detections and their confidence, and allows us to quickly examine and reject falsely detected sites. We determine a threshold parameter $b$ for the LE detector in (18) based on the number of allowed false detections. The user interface allows choosing the number of false detections to be generated for an analyzed image.

We applied our detector to the region of the Silvretta Alps [50] of about $550 \mathrm{~km}^{2}$ size. We used panchromatic images at $0.5-\mathrm{m}$ resolution captured by the GeoEye- 1 satellite. The data stem from a recent archaeological project in the Silvretta Alps [2]. We also repeated our experiments using the red channel of SWISSTOPO aerial images of $0.5-\mathrm{m}$ resolution that covered a slightly larger area of the same Silvretta region. However, the following technical details refer to the case of the satellite imagery.

The rectangularity feature may result in a large number of false detections in textured regions (e.g., forests). Since we are only interested in LEs that sparsely appear in grassland areas, high-contrast texture regions were filtered out using the morphological texture contrast (MTC) descriptor [31], [32], [51] thresholded with the Otsu method [52]. The size parameters $r_{1}$ and $r_{2}$ in the MTC were set to 30 and 60 pixels, respectively. This filters out urban areas, forests, rocky mountains, and 

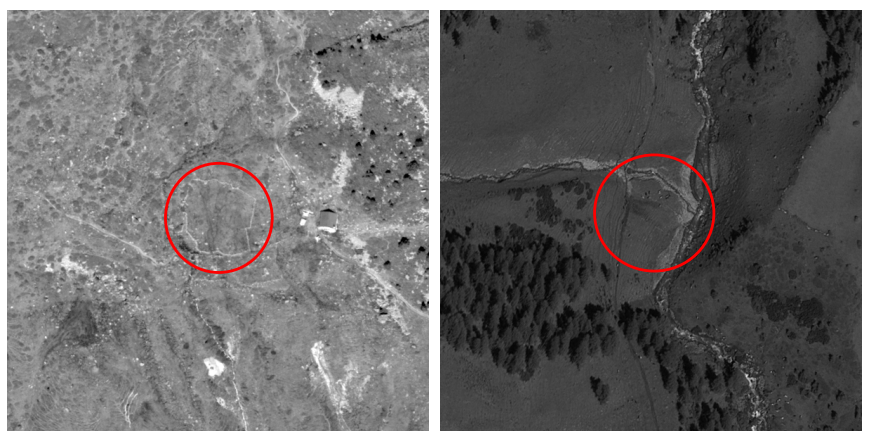

Fig. 8. (Left) Example of a previously unknown enclosure that was detected using the proposed rectangularity-size feature. (Right) Typical false detection.

other high-contrast texture regions, while preserving individual structures.

For learning the optimal direction $w_{\text {opt }}$, we used the 9 available examples of LEs and 49584 negative examples of structures around candidate points (see Section II) extracted from an $11000 \times 17000$ pixel satellite image. The detection threshold $b$ in (18) was set such that the number of generated detections was 5000 in the analyzed $550 \mathrm{~km}^{2}$ area. After visual inspection of detected sites with our user interface, we found 13 structures resembling LEs. Some of these detections were found to be LEs that were hitherto unknown. An example of such an enclosure is shown in Fig. 8 on the left. Fig. 8 on the right shows a typical false detection. False detections are usually caused by streams and roads. The use of 3-D data (e.g., LiDAR or based on stereo image pairs) would allow the discrimination of such false detections.

In our experiments, we used a MATLAB software. The satellite imagery that covers $550 \mathrm{~km}^{2}$ was divided into 17 partially overlapping images. Processing of all of the images (including all of the stages), which is equivalent to processing of a single $53000 \times 53000$ pixel image, took $3 \mathrm{~h}$ and $40 \mathrm{~min}$ on a machine with an Intel Core i5 3.3-GHz Quad-Core processor and 32-GB RAM.

\section{COMPARATIVE EXPERIMENTS}

\section{A. Features for Comparison}

We evaluated the discrimination ability of the introduced rectangularity feature $f_{R}$ and provided a comparison with the NMR measure $f_{\mathrm{NMR}}$ that we developed earlier in [20] and the GODF-based feature $f_{\mathrm{GODF}}$ in [10] recently proposed for building detection. The GODF, denoted $\lambda(\theta)$, is a weighted gradient orientation histogram with gradient magnitudes as weights and discrete orientation $\theta \in[0,180)$. The correlation of $\lambda(\theta)$ with a function having two modes separated by $90^{\circ}$ served as a GODF-based feature $f_{\text {GODF }}$ indicating the presence of rectilinear structures. The normalization constant was set such that $\lambda(\theta)$ is a unit vector, which gave us better results than for the normalization constant equal to the sum of the weights used in [10]. More implementation details can be found in [24]. Note that we did not compare the rectangularity feature with the whole approach developed in [10] because it is based on additional features not appropriate in the case of enclosures.
We have also tested other methods for building detection (e.g., [8] and [11]) applied to detection of LEs. Unfortunately, these methods completely failed to detect enclosures. Thus, a corresponding quantitative comparison cannot be made.

We used the learning framework described in Section IV in order to evaluate and compare the developed rectangularitysize features $f_{\mathrm{RS}}$, high-dimensional HOG feature vectors $f_{\mathrm{HOG}}$ [30], and deep convolutional neural networks (CNNs) [53], [54] generating the so-called deep features, denoted $f_{\mathrm{CNN}}$ with CNN substituted by the name of a particular CNN architecture. These deep features are neural activations generated by pretrained CNNs at some intermediate layer of the deep network. Usually, these features are extracted either from the last convolutional layer or from one of the following fully connected layers but before the final one. There is mounting evidence that such features generated by CNNs pretrained on a very large data set of labeled images have a sufficient representation power to perform recognition tasks on completely different types of target images. Several recent works successfully used deep features in conjunction with either a fully connected neural network [55] or even a simple linear classifier [56]-[58] trained on a relatively small target set of images. Moreover, deep features were also shown to be useful for classification of remotely sensed images [59]. Such an approach allows us to use CNNs even though we have a very limited amount of positive examples to learn from.

We generated deep features using several CNN models pretrained on the subsets of the ImageNet database [60]. Deep features $f_{\mathrm{Vgg}-\mathrm{f}}, f_{\mathrm{Vgg}-\mathrm{m}}, f_{\mathrm{Vgg}-\mathrm{m}-2048}, f_{\mathrm{Vgg}-\mathrm{s}}{ }^{6}$ were extracted from the CNN architectures described in [27]. $f_{\mathrm{Vgg} \text {-deep-16, }}$, $f_{\text {OverFeat }}$, and $f_{\text {GoogLeNet }}$ were extracted from the networks described in [26], [28], and [29], respectively. $f_{\text {AlexNet }}$ features were extracted from the network described in [25], while $f_{\text {CaffeNet }}$ features were generated by an independently trained variation of that network as mentioned in [61] and [62]. All deep features, except OverFeat, were computed using the MATLAB toolbox MatConvNet [63] using the pretrained models taken from the webpage [64] accompanying the toolbox. The OverFeat pretrained model was taken from the webpage [65], which has the source code implementing the deep network presented in [28]. The "fast" network was used. Following recommendations of the authors of the $\mathrm{CNN}$ models, except for OverFeat, we subtracted the mean image of the training data set from each image presented to the CNNs. Since we detect structures in grayscale images, while the CNN models require RGB channels as an input, we set each channel equal to the given grayscale image.

The HOG features were computed for $14 \times 14$ pixels cell size (with 7 pixels of overlap from one cell to the next) and 9 orientation bins, which gave us the best results among all configurations with which we experimented. We used the C source code of the HOG implementation available in the VLFeat package [66].

\footnotetext{
${ }^{6}$ We have also experimented with Vgg-m-1024 and Vgg-m-128 CNNs that have a smaller last hidden layer (1024 and 128 versus 4096 neurons in Vgg-m), but they gave worse results compared to Vgg-m. Therefore, we did not consider these features in our comparative experiments.
} 
All of the features were computed for image regions around the candidate points $p$. The size of these candidate regions was taken proportionally to the distance transform $D(p)$ (see Section II). To keep the size of HOG feature vectors constant, we resized the candidate regions to $160 \times 160$ pixel patches. Deep features were computed for the candidate regions resized to the size required by a particular $\mathrm{CNN}$ architecture.

\section{B. Measuring Discrimination Power of the Features}

Using a particular type of features $f$, LEs can be detected with $f>b$ for 1 -D features $\left(f_{R}, f_{\mathrm{NMR}}, f_{\mathrm{GODF}}\right)$ and with $f^{T} w_{\mathrm{opt}}>b$ for multidimensional features $\left(f_{\mathrm{RS}}, f_{\mathrm{HOG}}, f_{\mathrm{CNN}}\right)$, where $b$ is an appropriate threshold to be set. Setting a particular threshold defines the true positive rate (TPR) and the false positive rate (FPR) or correspondingly the number of detected true and false positives (TP and FP). In our case, the effectiveness of the features is their ability to discriminate LEs from irrelevant structures and clutter. A possible measure of this ability is the minimal number of FP detected with the threshold that ensures TPR $\geq \xi$, where $\xi$ is the predefined rate of true positives. ${ }^{7}$ We computed $F P$ for $\xi=1$, denoted in the following by $F P_{100}$. This was done by setting the detection threshold $b$ to the minimum value of $f$ for 1-D features and $f^{T} w_{\text {opt }}$ for multidimensional features computed for all positive examples. Obviously, the threshold used to obtain the detection rate TPR $=1$ on a small number of available examples does not ensure a detector with $100 \%$ detection rate. However, it allows us to measure and compare the discrimination ability of the features. $F P_{100}$ is related to the spread of the class of positives toward the samples of the class of negatives, similarly to the Fisher criterion of discrimination ability [47]. However, $F P_{100}$ also gives a rough estimate of the minimal number of false detections per area size that should be allowed in order to have a reasonable detection rate. Unfortunately, the actual detection rate cannot be reliably estimated due to a very small number of positive examples.

We also used an alternative measure of the discrimination ability that is the area under the receiver operating characteristic (ROC) curve. It is especially useful in the presence of unbalanced classes [68], [69]. In contrast to $F P_{100}$, the area under ROC $(A U C)$ does not rely on a particular threshold and a corresponding operating point on the ROC curve but instead summarizes the detection performance for different values of the threshold. We estimated $A U C$ using the Wilcoxon-Mann-Whitney statistic [69], [70].

\section{Evaluation Procedure}

In our experiments here, we used panchromatic satellite images at $0.5-\mathrm{m}$ resolution that cover mountainous regions of the Silvretta Alps. Similarly to Section V, high-contrast texture regions were filtered out using the MTC descriptor [31], [32], [51]. We generated 49584 negative samples for training. The samples were taken around candidate points in a $11000 \times$ 17000 pixel satellite image. For testing, we used 57504 negative

\footnotetext{
${ }^{7}$ This corresponds to the so-called Neyman-Pearson task [67].
}

TABLE I

COMPARISON OF DISCRIMINATION MEASURES FOR THE FEATURES $f_{\mathrm{NMR}}, f_{\mathrm{GODF}}$ AND THE PROPOSED FEATURES $f_{R}, f_{\mathrm{RS}}$

\begin{tabular}{r|rrr}
\hline & $F P_{100}$ & $A U C \times 10^{2}$ & $\operatorname{dim}$. \\
\hline$f_{\mathrm{GODF}}$ & $\mathbf{6 8 6 2}$ & 99.262 & 1 \\
$f_{\mathrm{NMR}}$ & $\mathbf{7 0 7}$ & 99.925 & 1 \\
$f_{\mathrm{R}}$ & $\mathbf{2 9 2}$ & 99.967 & 1 \\
$f_{\mathrm{RS}}$ & $\mathbf{2 0 1}$ & 99.977 & 2 \\
\hline
\end{tabular}

samples taken from a different satellite image of $10000 \times$ 17000 pixel size that covers about $42.5 \mathrm{~km}^{2}$. Overall, only nine examples of enclosures (positives) taken from aerial and satellite images were available to us. We augmented these data with additional 135 rotated versions of the same enclosures. Here, 16 rotation angles were taken uniformly in the interval $[0,360)$ degrees. This results in 144 positive examples, which is hardly enough for training and evaluation on separate train and test subsets as we have done with negative samples. In the case of high-dimensional feature vectors, the learned classifier parameters $w_{\mathrm{opt}}$ and the estimated performance may largely vary, depending on the selected subset of positives. In order to use most of the positives for training and also make reliable evaluation of the classier performance based on the data not used for training, we perform ninefold cross validation. Note that we do not have hyperparameters associated with the classifier that need to be set a priori or optimized on spare data. On each fold, we use 16 examples of the same enclosure at different angles for testing and other 128 positives for training.

In the following, we report the average value of the performance measures and the standard deviation over the nine folds of cross validation. In addition, we compare the sensitivity of $F P_{100}$ to the reduction in the number of positives used for training. To do so, we compute the "inverted" ninefold cross validation where, on each fold, we use only 16 examples of a single enclosure at different angles for training. For testing, we use all 144 positives on each fold. Thus, the results may vary only due to the used training data, since the same data set is used for the performance evaluation. Note that, for the case of $F P_{100}$ measure, using all of the positives for testing including a single training example can yield only higher (worse) $F P_{100}$ because the worst positive sample defines $F P_{100}$.

\section{Results}

The quantitative measures of the discrimination performance of the rectangularity $f_{R}$ and the rectangularity-size features $f_{\mathrm{RS}}$ are summarized in Table $\mathrm{I}$. The performance measures $F P_{100}$ and $A U C$ evaluate the discrimination ability of the features for our task. The $F P_{100}$ (see Section VI-B) measures the number of false detections obtained in an area of approximately $42.5 \mathrm{~km}^{2}$, when all available positives are detected. This measure is particularly useful as it helps to decide how many false detections should be allowed in order to have a high detection rate, i.e., the rate that ensures detection of at least all available positive examples. The $A U C$ measure yields a performance ranking of different feature types similar to that of $F P_{100}$. On the other hand, unlike $F P_{100}$, the absolute values of $A U C$ are quite close to each other, giving the wrong impression of similar performance. The high and close values of $A U C$ are due to the 
TABLE II

Comparison of Discrimination Measures for Multidimensional CNN, HOG, and the Proposed ReCtangularity-Size Features $f_{\text {RS }}$. Ninefold Cross Validation Was Performed on 144 (Augmented) Positives With Either 128 or 16 Samples ( $\left.N_{\text {pos }}\right)$ Used for Training. Large SeParate Data Sets of Negatives Were Used for Training and Testing. "fc" Adjacent to the Layer Number in the Table Stands For "Fully Connected"

\begin{tabular}{|c|c|c|c|c|c|c|c|c|c|}
\hline \multirow{3}{*}{ feature } & \multicolumn{6}{|c|}{$N_{\text {pos }}=128$} & \multirow{3}{*}{$\begin{array}{c}N_{\text {pos }}=16 \\
\begin{array}{c}F P_{100} \\
\text { mean }\end{array}\end{array}$} & \multirow{3}{*}{ dimensionality } & \multirow{3}{*}{ output layer } \\
\hline & \multicolumn{3}{|c|}{$F P_{100}$} & \multicolumn{3}{|c|}{$A U C \times 10^{2}$} & & & \\
\hline & iean & & $\max$ & mean & std & min & & & \\
\hline$f_{\mathrm{RS}}$ & 49.3 & 64.3 & 203 & 99.976 & 0.036 & 99.884 & 206.3 & 2 & \\
\hline$f_{\text {AlexNet }}$ & 178.6 & 373.7 & 1145 & 99.943 & 0.124 & 99.615 & 35834.1 & 4096 & $\mathrm{fc}_{7}$ \\
\hline$f_{\mathrm{Vgg}-\mathrm{f}}$ & 195.4 & 269.5 & 847 & 99.849 & 0.213 & 99.358 & 22699.8 & 4096 & $\mathrm{fc}_{6}$ \\
\hline$f_{\text {Vgg-m-2048 }}$ & 365.8 & 520.6 & 1688 & 99.814 & 0.287 & 99.091 & 25895.0 & 4096 & $\mathrm{fc}_{6}$ \\
\hline$f_{\text {Vgg-deep-19 }}$ & 578.1 & 1694.3 & 5096 & 99.718 & 0.826 & 97.515 & 32573.9 & 4096 & $\mathrm{fc}_{17}$ \\
\hline$f_{\text {CaffeNet }}$ & 609.7 & 1153.8 & 3498 & 99.771 & 0.384 & 99.000 & 46673.3 & 4096 & $\mathrm{fc}_{6}$ \\
\hline$f_{\text {Vgg-m }}$ & 631.3 & 1039.9 & 2600 & 99.752 & 0.484 & 98.527 & 31120.2 & 4096 & $\mathrm{fc}_{6}$ \\
\hline$f_{\text {GoogLeNet }}$ & 1002.4 & 2176.5 & 6549 & 99.727 & 0.608 & 98.161 & 18603.1 & 1024 & avg $\operatorname{pool}_{21}$ \\
\hline$f_{\mathrm{Vgg-s}}$ & 2911.2 & 7097.7 & 21720 & 99.353 & 1.348 & 95.835 & 38231.3 & 4096 & $\mathrm{fc}_{6}$ \\
\hline$f_{\text {OverFeat }}$ & 2967.3 & 8164.2 & 24715 & 99.416 & 1.554 & 95.281 & 37536.7 & 3072 & $\mathrm{fc}_{6}$ \\
\hline$f_{\text {Vgg-deep-16 }}$ & 3473.0 & 10294.5 & 30925 & 99.447 & 1.576 & 95.244 & 39626.8 & 4096 & $\mathrm{fc}_{14}$ \\
\hline$f_{\mathrm{HOG}}$ & 7472.8 & 8352.8 & 27155 & 97.926 & 2.404 & 92.045 & 54154.9 & 4356 & \\
\hline$f_{\text {Rand-4096 }}$ & 54039.1 & 3124.5 & 57019.9 & 49.871 & 6.694 & 39.629 & 57054.1 & 4096 & \\
\hline$f_{\text {Rand-2 }}$ & 54227.0 & 2819.5 & 57087.5 & 50.736 & 6.164 & 41.098 & 57034.4 & 2 & \\
\hline
\end{tabular}

ability of the detectors to reject most negatives while detecting a modest number of all available positives. The corresponding ROCs saturate at the maximum detection rate already for small values of FPR and differ only for lower FPRs. Nevertheless, along with the $F P_{100}$, which is more intuitive and useful for our application, we also provide $A U C$ because it is commonly used for evaluation of detector performance. The last column in Table I indicates the dimensionality of the features.

Table I shows that the discrimination ability of the rectangularity-size features $f_{\mathrm{RS}}$ is superior to the others. It allowed reduction of false positives by $31 \%$ relative to $f_{R}$, which is, in turn, considerably better than the NMR measure. Although effective for building detection, the GODF-based feature turned out to be far worse for detecting faint enclosures in cluttered background. This feature is not useful when computed over large windows, where the relative number of points belonging to an enclosure is small.

To compute the rectangularity-size features $f_{\mathrm{RS}}$, we learned $w_{\text {opt }}$ from the separate training data set of 49584 negative examples (see Section VI-C). The set of 144 augmented positives used for testing of all of the feature types was also used for training the linear classifier. Learning the 2-D $w_{\text {opt }}$ involves positives only via their average $\bar{y}$ [see (17)] and uses separate large data sets of negatives; therefore, it is unlikely that the data are overfitted. Nevertheless, in the following, we carried out another set of experiments, where we avoid the use of the same positives for training and testing by means of the cross validation procedure (see Section VI-C for details). Using cross validation also allowed us comparison with high-dimensional HOG and deep-CNN-based features (deep features), which are much harder to keep from overfitting.

Table II shows the discrimination performance of the rectangularity-size features $f_{\mathrm{RS}}$, high-dimensional HOG $f_{\mathrm{HOG}}$, and deep features $f_{\text {CNN }}$ generated by several pretrained CNNs. For all CNN architectures, the table gives the layer used to extract features that produced the best result. Given a particular set of features, we use the methodology described in Section IV based on training the linear classifier (learning the hyperplane $w_{\mathrm{opt}}$ ). The table shows the mean values, standard deviation, and worse values ( $\max$ or $\min$ ) for the discrimination measures $F P_{100}$ and $A U C$ over nine folds of cross validation. On each fold of cross validation, 128 positives (augmented from 8 real examples) were used for training, and the remaining 16 positives (augmented from the ninth remaining example) were used for testing (see Section VI-C for details). The mean values of $F P_{100}$ show that the rectangularity-size features $f_{\mathrm{RS}}$ outperform all of the other features by a large margin. Surprisingly, however, two architectures of CNN, AlexNet [25] and Vgg-f [27], provided us with deep features that showed relatively high performance. This is a remarkable result because the CNNs were trained on a completely different image data set, while the linear classifier $w_{\text {opt }}$ was trained on 128 examples augmented from just 8 real enclosures. Note that no finetuning of the pretrained $\mathrm{CNN}$ was performed. These results also indicate that the simple methodology that we developed in Section IV for learning from a few positive and a large number of negative examples is useful even for the case of highdimensional features, although, as we show in the following, the performance of such features is much more sensitive to the number of positives used for training the linear classifier.

We experimented with CNNs with one or two final fully connected layers or the softmax function of the last layer removed. The table shows the layer that yields the best performing features. For AlexNet, the best result was obtained when two final fully connected layers were kept. For all of the other CNNs, the best results were obtained when features were taken from the first layer that generates data reduced to $1 \times 1$ spatial dimension, which is an average pooling for the GoogLeNet and a fully connected layer for all of the other CNNs. Our results support the hypothesis that convolutional layers of pretrained CNNs generate generic features that might be useful for various tasks. In contrast, the final fully connected layers generate taskspecific features.

Table II also shows how the performance for all features dropped when only 16 augmented samples were used for training (see Section VI-C for details) within each fold of 


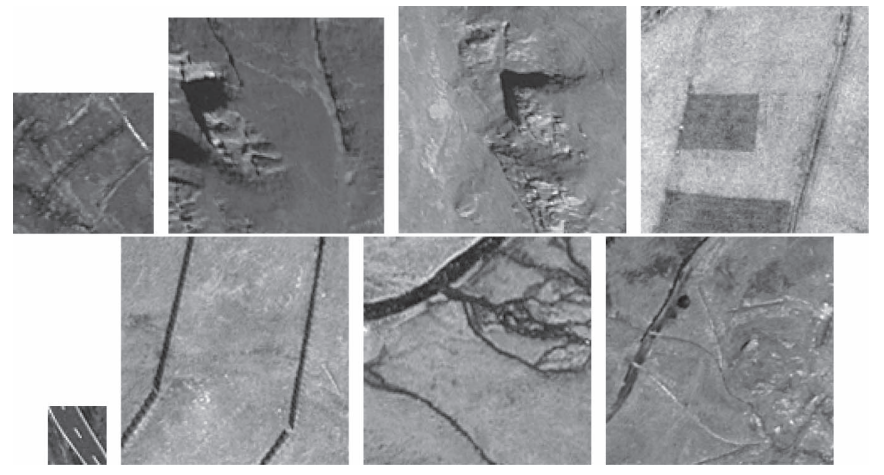

Fig. 9. Candidate patches generating the highest responses by AlexNet (top row) and Vgg-f (bottom row) architectures of pretrained CNNs followed by a linear classifier trained on 8 real examples (128 augmented examples) of rectangular enclosures and large number of negatives. Note that these patches contain structures that are conceptually close to rectangles.

cross validation. However, in contrast to the other features, the rectangularity-size $f_{\mathrm{RS}}$ still yielded relatively high performance, while all of the other high-dimensional features became not useful. This experiment showed high sensitivity of the performance to the number of training examples for the case of high-dimensional features. This also suggests that collecting additional examples might substantially improve their performance.

We also notice that the deeper architectures (Vgg-deep-16, Vgg-deep-19, and GoogLeNet) did not have superior performance for our task. The architecture of CNNs was more important than just their depth, which is in line with a recent observation in [71]. Note that the best performing CNNs AlexNet and Vgg-f have similar architectures [27]. The importance of the particular architecture is also evident from the large variability of the performance of the different CNNs in Table II. Moreover, although CaffeNet and AlexNet are supposed to perform similarly (the first network is a minor variation of the second [62]), they produce substantially different results. The differences in particular training procedures may be responsible for such a discrepancy. The choice of the CNN architecture and training procedure was crucial for our task and seems likely to be critical for other applications. However, it seems that currently there is no established alternative to the trial-and-error based choice of the most suitable architecture for the task at hand.

In Fig. 9, we show candidate patches seen by AlexNet and Vgg-f CNNs that generate top responses of the linear classifier $f_{\mathrm{CNN}}^{T} w_{\text {opt }}$. The patches were taken out of 57504 negative samples used for testing. These top response patches resemble the structures of our interest, indicating that corresponding deep features might be powerful enough to capture the concept of the rectangular enclosures.

For reference purposes, in Table II, we also evaluate the performance of random feature vectors $f_{\text {Rand }}$ using the same evaluation strategy. The random feature vectors with 2 and 4096 entries of independently identically distributed variables were drawn from the standard normal distribution. As expected, such features give average $A U C$ values close to 0.5 . The resulting mean values for false positives $F P_{100}$ are not far from the overall number of samples used for testing (57504).

\section{Application to Detection of Buildings}

Although the rectangularity feature was developed for a particular task of detecting ruined LEs, it can also be used for other tasks. Here, we illustrate its application to building detection. Since the MFC-based line detector extracts bar edges only, we replaced it with the line segment detector of [36] that also extracts step edges that are more appropriate for detection of buildings. In the case of strong object contrasts (unlike the case of LEs), it reliably detects object borders and yields relatively small number of edges caused by clutter.

Once the edges were extracted, we used the same algorithms as described in Sections II and III in order to generate the rectangularity feature $f_{R}$. Since we do not have a labeled training data set for buildings, we did not experiment here with the rectangularity-size features $f_{\mathrm{RS}}$. We also do not expect that it can essentially be better than $f_{R}$ because the variability of building sizes is much smaller than that for the case of the enclosures. Nevertheless, we still took into account the dependence of the $f_{R}$ on the size of the structure by normalizing the rectangularity feature as $f_{R} / f_{S}$. Fig. 10 illustrates $\ln \left(f_{R} / f_{S}\right)$ computed for a SWISSTOPO $4000 \times 4000$ aerial image of $0.25-\mathrm{m}$ resolution taken over the Bernese Alps. The logarithm was taken in order to make weak detections better visible. We used the same parameters as before for $0.5-\mathrm{m}$ resolution images, except for the maximal size of building structures. The maximal size was reduced to 65 pixels, while the minimal size was kept to 15 pixels. One can see that most of the buildings were detected. On the other hand, there are false detections mostly caused by occasional configurations of forest and field edges or roads. In urban areas, our detector may produce many false detections in between adjacent buildings or other manmade structures. Therefore, in the original form, the detector might be more appropriate for rural or mountainous areas when high sensitivity is needed for detection of possibly occluded individual rare structures. For better performance, it can be adapted to detect buildings (instead of enclosures) by, for example, incorporating region and/or corner cues. Such adaptations, however, are out of the scope of this paper, as is the quantitative evaluation of performance for building detection tasks.

\section{CONCLUSION}

We have introduced the scalar rectangularity feature $f_{R}$ for detection of approximately rectangular livestock enclosure structures in remotely sensed imagery. It has shown high performance in discriminating ruined enclosures from irrelevant structures and clutter. Due to the inherent difficulties of our problem, such a performance is hardly achievable with other approaches for detection of rectangular contours, nor with related approaches, e.g., for detection of buildings. Note that, while the building detection problem can be addressed using the enclosure detector (see Section VII for example), detection of enclosures cannot be approached using building detectors. In general, methods for building detection are not suitable for our case because of considerably lower heights (resulting in low feature contrasts) and feature sizes (ruined walls versus building rooftops) and due to the absence of various cues (roof colors, roof homogeneity, shadows, 3-D cues, etc.). Some walls or parts 

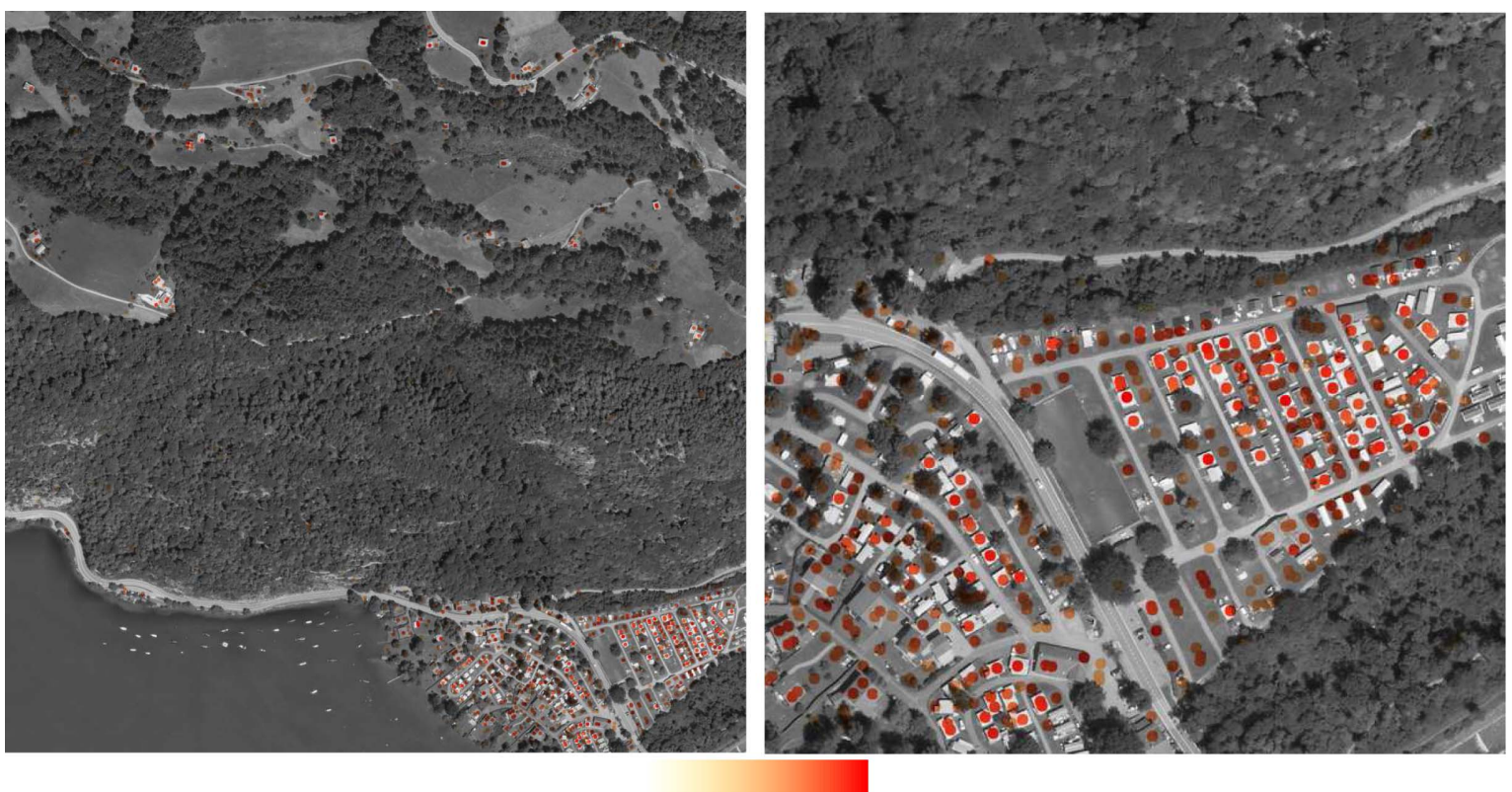

Fig. 10. (Left) Building detections in a $4000 \times 4000$ aerial (SWISSTOPO) image of $0.25-\mathrm{m}$ resolution visualized by colored disks. (Right) Enlarged bottom right part of the image in the left. Color saturation increases, and hue is changing from yellow to red for growing values of $\ln \left(f_{R} / f_{S}\right)$ in accordance with the color bar at the bottom.

of them may be missing or may also be missed in the edge extraction (the width of linear features does not exceed two pixels in images of $0.5-\mathrm{m}$ resolution). Various irrelevant structures (trails, streams, rocks, etc.) with sizes or/and reflectance properties similar to those of enclosure walls may occasionally form rectilinear configurations. In contrast to enclosures, building rooftops are much more distinctive structures. As an example, we have shown that the GODF-based feature used for detection of buildings reveals a poor discrimination ability for our task.

We have also designed a size feature and introduced a methodology used for learning a linear classifier in the 2-D rectangularity-size feature space that improves over the detector based solely on the rectangularity feature. The same methodology in high-dimensional feature space was used to learn the classifier based on HOG feature vectors and feature vectors generated by pretrained deep CNNs (deep features). Quantitative comparison has shown that the rectangularity-size features $f_{\mathrm{RS}}$ clearly outperform these state-of-the-art features for our task.

However, we have found that several pretrained deep CNN architectures (that yield generic deep features) along with the linear classifier trained using our methodology from just a few positive samples (augmented using rotations) and a large number of negatives may still result in a well-performing detector. Although these CNN-based features did not perform as well as the rectangularity-size features $f_{\mathrm{RS}}$, they may still be useful for detection of the LEs of very low contrasts. Contrary to $f_{\mathrm{RS}}$, they do not require a separate stage of extracting bar edges, which may fail in the cases of very low contrasts (e.g., due to low heights of ruined walls). Moreover, given examples of enclosures of nonrectangular shape, we could easily retrain our linear classifier using the same generic deep features. The resulting performance is likely to be improved by learning from more augmented examples using additional transformations, e.g., scaling, flipping, brightness transformations, etc. Availability of additional (real) positive examples is certainly critical for improving performance of the deep-CNN-based detector and may also enable performing fine-tuning of the CNN itself for further gain in performance. The aforementioned issues are interesting for future research.

We have reported that, by using our algorithms (detection of bar edges, candidate generation, computation of the rectangularity-size features, and linear classification), we have detected LEs in the Silvretta Alps that were hitherto unknown. We have also discussed a different application of the rectangularity feature for detection of buildings in rural or mountainous areas. For better performance in such an application, the rectangularity feature should be accompanied by other features capturing additional properties of buildings.

\section{ACKNOWLEDGMENT}

I. Zingman would like to thank the German Research Foundation (DFG) for the financial support within project A05 of SFB/Transregio 161. O. A. B. Penatti would like to thank the RECOD Laboratory, University of Campinas, Campinas, Brazil, for the infrastructure used for the computation of the HOG and OverFeat features.

\section{REFERENCES}

[1] K. Kothieringer et al., "High impact: Early pastoralism and environmental change during the Neolithic and Bronze Age in the Silvretta Alps (Switzerland/Austria) as evidenced by archaeological, palaeoecological and pedological proxies," Zeitschrift Geomorphologie, vol. 59, no. 2, pp. 177-198, 2015.

[2] K. Lambers and I. Zingman, "Towards detection of archaeological objects in high-resolution remotely sensed images: The Silvretta case study," in Archaeology in the Digital Era, vol. II (e-papers) From the 40th Conf. on Computer Applications and Quantitative Methods in Archaeology, 
Southampton, March 2012, G. Earl et al., Eds. Amsterdam, The Netherlands: Amsterdam Univ. Press, 2013, pp. 781-791.

[3] Ø. D. Trier, S. Ø. Larsen, and R. Solberg, "Automatic detection of circular structures in high-resolution satellite images of agricultural land," Archaeol. Prospection, vol. 16, pp. 1-15, 2009.

[4] H. Mayer, "Automatic object extraction from aerial imagery-A survey focusing on buildings," Comput. Vis. Image Understand., vol. 74, no. 2, pp. 138-149, 1999.

[5] C. Lin and R. Nevatia, "Building detection and description from a single intensity image," Comput. Vis. Image Understand., vol. 72, no. 2, pp. 101-121, 1998.

[6] T. Kim and J.-P. Muller, "Development of a graph-based approach for building detection," Image Vis. Comput., vol. 17, no. 1, pp. 3-14, 1999.

[7] A. Croitoru and Y. Doytsher, "Right-angle rooftop polygon extraction in regularised urban areas: Cutting the corners," Photogramm. Rec., vol. 19, no. 108 , pp. 311-341, 2004.

[8] C. R. Jung and R. Schramm, "Rectangle detection based on a windowed Hough transform," in Proc. 17th Brazilian SIBGRAPI, 2004, pp. 113-120.

[9] S. Krishnamachari and R. Chellappa, "Delineating buildings by grouping lines with MRFs," IEEE Trans. Image Process., vol. 5, no. 1, pp. 164-168, Jan. 1996.

[10] C. Benedek, X. Descombes, and J. Zerubia, "Building development monitoring in multitemporal remotely sensed image pairs with stochastic birth-death dynamics," IEEE Trans. Pattern Anal. Mach. Intell., vol. 34, no. 1, pp. 33-50, Jan. 2012.

[11] B. Sirmacek and C. Unsalan, "A probabilistic framework to detect buildings in aerial and satellite images," IEEE Trans. Geosci. Remote Sens., vol. 49, no. 1, pp. 211-221, Jan. 2011.

[12] B. Sirmacek and C. Unsalan, "Urban-area and building detection using SIFT keypoints and graph theory," IEEE Trans. Geosci. Remote Sens., vol. 47, no. 4, pp. 1156-1167, Apr. 2009.

[13] A. Manno-Kovacs and T. Sziranyi, "Multidirectional building detection in aerial images without shape templates," Int. Archives Photogramm., Remote Sens. Spatial Inf. Sci., vol. XL-1/W1, pp. 227-232, May 2013.

[14] M. Ortner, X. Descombes, and J. Zerubia, "A marked point process of rectangles and segments for automatic analysis of digital elevation models," IEEE Trans. Pattern Anal. Mach. Intell., vol. 30, no. 1, pp. 105-119, Jan. 2008.

[15] Y. Liu, T. Ikenaga, and S. Goto, "An MRF model-based approach to the detection of rectangular shape objects in color images," Signal Process., vol. 87, no. 11, pp. 2649-2658, 2007.

[16] C. G. Keller, C. Sprunk, C. Bahlmann, J. Giebel, and G. Baratoff, "Realtime recognition of US speed signs," in Proc. IEEE Intell. Veh. Symp., 2008, pp. 518-523.

[17] G. B. Loy and N. M. Barnes, "Fast shape-based road sign detection for a driver assistance system," in Proc. IEEE/RSJ Int. Conf. Intell. Robots Syst., Sendai, Japan, 2004, pp. 70-75.

[18] Y. Zhu, B. Carragher, F. Mouche, and C. S. Potter, "Automatic particle detection through efficient Hough transforms," IEEE Trans. Med. Imag., vol. 22, no. 9, pp. 1053-1062, Sep. 2003.

[19] Z. Yu and C. Bajaj, "Detecting circular and rectangular particles based on geometric feature detection in electron micrographs," J. Struct. Biol., vol. 145 , no. 1/2, pp. 168-180, 2004.

[20] I. Zingman, D. Saupe, and K. Lambers, "Automated search for livestock enclosures of rectangular shape in remotely sensed imagery," in Proc. 19th SPIE, Image Signal Process. Remote Sens., L. Bruzzone, Ed., Dresden, Germany, 2013, vol. 8892, pp. 1-11.

[21] H. Moon, R. Chellappa, and A. Rosenfeld, "Optimal edge-based shape detection," IEEE Trans. Image Process., vol. 11, no. 11, pp. 1209-1227, Nov. 2002.

[22] X. Descombes and J. Zerubia, "Marked point process in image analysis," IEEE Signal Process. Mag., vol. 19, no. 5, pp. 77-84, Sep. 2002.

[23] Y. Verdie and F. Lafarge, "Detecting parametric objects in large scenes by Monte Carlo sampling," Int. J. Comput. Vis., vol. 106, no. 1, pp. 57-75, 2014.

[24] I. Zingman, D. Saupe, and K. Lambers, "Detection of incomplete enclosures of rectangular shape in remotely sensed images," in Proc. IEEE Conf. CVPR Workshops, Jun. 2015, pp. 87-96.

[25] A. Krizhevsky, I. Sutskever, and G. E. Hinton, "ImageNet classification with deep convolutional neural networks," in Proc. Adv. Neural Inf. Process. Syst., 2012, pp. 1097-1105.

[26] K. Simonyan and A. Zisserman, "Very deep convolutional networks for large-scale image recognition," presented at the Int. Conf. Learning Representations (ICLR), May 2015.
[27] K. Chatfield, K. Simonyan, A. Vedaldi, and A. Zisserman, "Return of the devil in the details: Delving deep into convolutional nets," in Proc. Brit. Mach. Vis. Conf., 2014, p. 1.

[28] P. Sermanet et al., "OverFeat: Integrated recognition, localization and detection using convolutional networks," in Proc. ICLR, Apr. 2014, pp. 1-16.

[29] C. Szegedy et al., "Going deeper with convolutions," in Proc. IEEE CVPR, Jun. 2015, p. 1.

[30] N. Dalal and B. Triggs, "Histograms of oriented gradients for human detection," in Proc. IEEE Conf. CVPR, 2005, pp. 886-893.

[31] I. Zingman, D. Saupe, and K. Lambers, "A morphological approach for distinguishing texture and individual features in images," Pattern Recognit. Lett., vol. 47, pp. 129-138, Oct. 2014.

[32] I. Zingman, D. Saupe, and K. Lambers, "Detection of texture and isolated features using alternating morphological filters," in Mathematical Morphology and Its Applications to Signal and Processing, vol. 7883, ser. Lecture Notes in Computer Science, C. Hendriks, G. Borgefors, and R. Strand, Eds. New York, NY, USA: Springer-Verlag, 2013, pp. 440-451.

[33] T. Lindeberg, "Edge detection and ridge detection with automatic scale selection," Int. J. Comput. Vis., vol. 30, no. 2, pp. 117-156, 1998.

[34] C. Grigorescu, N. Petkov, and M. Westenberg, "Contour and boundary detection improved by surround suppression of texture edges," Image Vis. Comput., vol. 22, pp. 609-622, 2004.

[35] G. Papari and N. Petkov, "An improved model for surround suppression by steerable filters and multilevel inhibition with application to contour detection," Pattern Recognit., vol. 44, pp. 1999-2007, 2011.

[36] R. Grompone von Gioi, J. Jakubowicz, J.-M. Morel, and G. Randall, "LSD: A fast line segment detector with a false detection control," IEEE Trans. Pattern Anal. Mach. Intell., vol. 32, no. 4, pp. 722-732, Apr. 2010.

[37] K. Siddiqi, S. Bouix, A. Tannenbaum, and S. W. Zucker, "Hamilton-Jacobi skeletons," Int. J. Comput. Vis., vol. 48, no. 3, pp. 215-231, 2002.

[38] S. M. Pizer, K. Siddiqi, G. Székely, J. N. Damon, and S. W. Zucker, "Multiscale medial loci and their properties," Int. J. Comput. Vis., vol. 55, no. 2/3, pp. 155-179, 2003.

[39] P. Dimitrov, J. N. Damon, and K. Siddiqi, "Flux invariants for shape," in Proc. IEEE Conf. CVPR, 2003, vol. 1, p. I-835.

[40] D. Engel and C. Curio, "Scale-invariant medial features based on gradient vector flow fields," in Proc. ICPR, 2008, pp. 1-4.

[41] C. Xu and J. L. Prince, "Snakes, shapes, and gradient vector flow," IEEE Trans. Image Process., vol. 7, no. 3, pp. 359-369, Mar. 1998.

[42] C. Xu and J. L. Prince, "Generalized gradient vector flow external forces for active contours," Signal Process., vol. 71, no. 2, pp. 131-139, 1998.

[43] R. O. Duda and P. E. Hart, "Use of the Hough transformation to detect lines and curves in pictures," Commun. ACM, vol. 15, no. 1, pp. 11-15, Jan. 1972.

[44] L. Lam, S.-W. Lee, and C. Y. Suen, "Thinning methodologies-A comprehensive survey," IEEE Trans. Pattern Anal. Mach. Intell., vol. 14 no. 9, pp. 869-885, Sep. 1992.

[45] R. O. Duda and P. E. Hart, Pattern Classification and Scene Analysis. Oxford, U.K.: Wiley, 1973.

[46] C. Bron and J. Kerbosch, "Algorithm 457: Finding all cliques of an undirected graph," Commun. ACM, vol. 16, no. 9, pp. 575-577, Sep. 1973.

[47] K. Fukunaga, Introductionto Statistical Pattern Recognition. New York, NY, USA: Academic, 1990.

[48] S. J. Devlin, R. Gnanadesikan, and J. R. Kettenring, "Robust estimation of dispersion matrices and principal components," J. Amer. Statist. Assoc., vol. 76, no. 374, pp. 354-362, 1981.

[49] B. Hariharan, J. Malik, and D. Ramanan, "Discriminative decorrelation for clustering and classification," in Proc. Eur. Conf. Comput. Vis., 2012, pp. 459-472.

[50] K. Lambers and T. Reitmaier, "Silvretta Historica: Satellite-assisted archaeological survey in an alpine environment," in CAA 2010 Fusion of Cultures: Proceedings of the 38th Annual Conference on Computer Applications and Quantitative Methods in Archaeology, Granada, Spain, April 2010, F. Contreras, M. Farjas, and F. J. Melero, Eds. Oxford, U.K.: Archaeopress, 2013.

[51] I. Zingman, D. Saupe, and K. Lambers, "Morphological operators for segmentation of high contrast textured regions in remotely sensed imagery," in Proc. IEEE Int. Geosci. Remote Sens. Symp., Munich, Germany, Jul. 2012, pp. 3451-3454.

[52] N. Otsu, "A threshold selection method from gray-level histograms," IEEE Trans. Syst., Man Cybern., vol. 9, no. 1, pp. 62-66, Jan. 1979. 
[53] S. Haykin, Neural Networks and Learning Machines, 3rd ed. London, U.K.: Pearson, 2009, ch. 4.17.

[54] Y. LeCun, Y. Bengio, and G. Hinton, "Deep learning," Nature, vol. 521, no. 7553, pp. 436-444, 2015.

[55] M. Oquab, L. Bottou, I. Laptev, and J. Sivic, "Learning and transferring mid-level image representations using convolutional neural networks," in Proc. IEEE Conf. CVPR, Jun. 2014, pp. 1717-1724.

[56] J. Donahue et al., "DeCAF: A deep convolutional activation feature for generic visual recognition," in Proc. 31st ICML, Jun. 2014, pp. 647-655.

[57] A. S. Razavian, H. Azizpour, J. Sullivan, and S. Carlsson, "CNN features off-the-shelf: An astounding baseline for recognition," in Proc. IEEE CVPRW, 2014, pp. 512-519.

[58] R. Girshick, J. Donahue, T. Darrell, and J. Malik, "Region-based convolutional networks for accurate object detection and segmentation," IEEE Trans. Pattern Anal. Mach. Intell., vol. 38, no. 1, pp. 142-158, Jan. 2015.

[59] O. A. B. Penatti, K. Nogueira, and J. A. dos Santos, "Do deep features generalize from everyday objects to remote sensing and aerial scenes domains?" in Proc. IEEE CVPRW, 2015, pp. 44-51.

[60] O. Russakovsky et al., "ImageNet large scale visual recognition challenge," in Proc. IJCV, Apr. 2015, pp. 1-42.

[61] Y. Jia et al., "Caffe: Convolutional architecture for fast feature embedding," in Proc. 22nd ACM Int. Conf. Multimedia, 2014, pp. 675-678.

[62] Caffe Model Zoo. [Online]. Available: http://caffe.berkeleyvision.org/ model_zoo

[63] A. Vedaldi and K. Lenc, "MatConvNet-Convolutional neural networks for MATLAB," in Proc. ACM Int. Conf. Multimedia, 2015, pp. 689-692.

[64] Pre-Trained CNN Models. Accessed: Nov. 18, 2015. [Online]. Available: http://www.vlfeat.org/matconvnet/pretrained

[65] Pre-Trained OverFeat. Version: v04-2. [Online]. Available: https://github. com/sermanet/OverFeat

[66] A. Vedaldi and B. Fulkerson, "VLFeat: An open and portable library of computer vision algorithms," in Proc. 18th ACM Int. Conf. Multimedia. Version: 0.9.16. [Online]. Available: http://www.vlfeat.org/

[67] M. I. Schlesinger and V. Hlavac, Ten Lectures on Statistical and Structural Pattern Recognition. Berlin, Germany: Springer-Verlag, 2002.

[68] T. Fawcett, "An introduction to ROC analysis," Pattern Recognit. Lett., vol. 27, no. 8, pp. 861-874, Jun. 2006.

[69] W. J. Krzanowski and D. J. Hand, ROC Curves for Continuous Data. London, U.K.: Chapman \& Hall, 2009.

[70] J. A. Hanley and B. J. McNeil, "The meaning and use of the area under a receiver operating characteristic (ROC) curve," Radiology, vol. 143, no. 1, pp. 29-36, 1982.

[71] B. Pepik, R. Benenson, T. Ritschel, and B. Schiele, "What is holding back ConvNets for detection?" in Pattern Recognition. Berlin, Germany: Springer-Verlag, 2015, pp. 517-528.

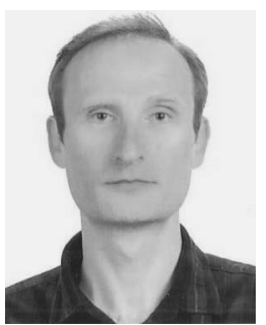

learning.

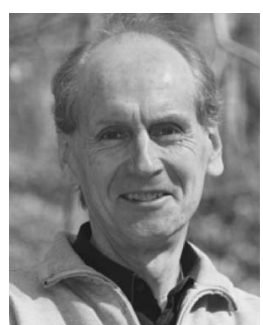

Dietmar Saupe received the Doctorate and Habilitation degrees in mathematics from the University of Bremen, Bremen, Germany.

$\mathrm{He}$ is a Professor of computer science with the University of Konstanz, Konstanz, Germany. His previous professorships were at the Department of Mathematics, University of California, Santa Cruz, CA, USA, in 1985-1987 and then as a Professor of computer science with the University of Freiburg, Freiburg, Germany, in 1993-1998 and University of Leipzig, Leipzig, Germany, in 1998-2002. He is the coauthor of several popular books on chaos and fractals. His current areas of interest include multimedia signal processing, sport informatics, and image/video processing.

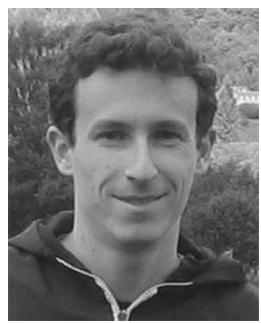

Otávio A. B. Penatti received the M.Sc. and Ph.D. degrees in computer science from the University of Campinas, Campinas, Brazil, in 2009 and 2012, respectively.

He is a Research Scientist with the Samsung Research Institute, Campinas. His research interests include computer vision, pattern recognition, machine learning, content-based image and video retrieval, and multimedia geocoding.

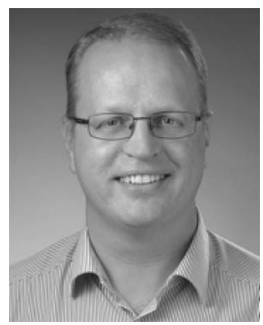

Karsten Lambers received the Master's degree in American anthropology from the University of Bonn, Bonn, Germany, in 1998 and the Ph.D. degree in prehistoric archaeology from the University of Zurich, Zurich, Switzerland, in 2005.

$\mathrm{He}$ is an Assistant Professor of archaeological computer sciences with the Faculty of Archaeology, Leiden University, Leiden, The Netherlands. He has held research positions at ETH Zurich (1999-2004), the University of Zurich (2004), the German Archaeological Institute (2005-2007), and the University of Konstanz (2008-2010) and a teaching position at the University of Bamberg (2010-2015). His main research interests revolve around computational methods in archaeology, with a focus on remote sensing, digital image analysis, and computer vision applied to archaeological prospection.

Dr. Lambers is a member of the steering committees of Computer Applications and Quantitative Methods in Archaeology (CAA) and of Archaeolandscapes International (ArcLand). 